\title{
Getting a grip on sensorimotor effects in lexical-semantic processing
}

\author{
Alison Heard ${ }^{1} \cdot$ Christopher R. Madan $^{2} \cdot$ Andrea B. Protzner $^{1} \cdot$ Penny M. Pexman ${ }^{1,3}$ \\ Published online: 2 July 2018 \\ (C) Psychonomic Society, Inc. 2018
}

\begin{abstract}
One of the strategies that researchers have used to investigate the role of sensorimotor information in lexical-semantic processing is to examine the effects of words' rated body-object interaction (BOI; i.e., the ease with which the human body can interact with a word's referent). Processing tends to be facilitated for words with high as compared with low BOI, across a wide variety of tasks. Such effects have been referenced in debates over the nature of semantic representations, but their theoretical import has been limited by the fact that BOI is a fairly coarse measure of sensorimotor experience with words' referents. In the present study, we collected ratings for 621 words on seven semantic dimensions (graspability, ease of pantomime, number of actions, animacy, size, danger, and usefulness), in order to investigate which attributes are most strongly related to BOI ratings and to lexicalsemantic processing. BOI ratings were obtained from previous norming studies (Bennett, Burnett, Siakaluk, \& Pexman in Behavior Research Methods, 43, 1100-1109, 2011; Tillotson, Siakaluk, \& Pexman in Behavior Research Methods, 40, 10751078, 2008), and measures of lexical-semantic processing were obtained from previous behavioral megastudies involving either the semantic categorization task (concrete/abstract decision; Pexman, Heard, Lloyd, \& Yap in Behavior Research Methods, 49, 407-417, 2017) or the lexical decision task (Balota et al., Behavior Research Methods, 39, 445-459, 2007). The results showed that the motor dimensions of graspability, ease of pantomime, and number of actions were all related to BOI, and that these dimensions together explained more variance in semantic processing than did the BOI ratings alone. These ratings will be useful for researchers who wish to study how different kinds of bodily interactions influence lexical-semantic processing and cognition.
\end{abstract}

Keywords Body-object interaction $\cdot$ Semantic decision task $\cdot$ Lexical decision task $\cdot$ Motor ratings $\cdot$ Visual word recognition

\section{Introduction}

In recent years, sensorimotor effects in lexical-semantic processing have been a vibrant topic of research. This interest has been driven largely by the relevance of these effects to theories of semantic representation. In particular, embodied semantic theories claim that retrieval of word meaning, even in simple reading and semantic decision tasks, involves activation of the sensorimotor and perceptual systems (e.g., Barsalou, 2008; Glenberg, 2015). As such, sensorimotor effects in lexical-semantic processing have been used to test the viability of embodied accounts of semantic representation.

Penny M. Pexman

pexman@ucalgary.ca

1 Department of Psychology and Hotchkiss Brain Institute, University of Calgary, Calgary, Alberta, Canada

2 School of Psychology, University of Nottingham, Nottingham, UK

3 Department of Psychology, University of Calgary, 2500 University Drive NW, Calgary, Alberta T2N1N4, Canada
One common strategy researchers have used to study sensorimotor effects in lexical processing is to examine effects of words' rated body-object interaction (BOI). BOI has been a focus of study because the dimension captures the relative availability of sensorimotor information (Hargreaves et al., 2012). Ratings of BOI are intended to measure the ease with which the human body can interact with a word's referent (Siakaluk, Pexman, Aguilera, Owen, \& Sears, 2008). Words whose referents afford relatively more BOI (e.g., toothbrush) are typically processed faster than words whose referents afford fewer opportunities for interaction (e.g., elephant) (Siakaluk, Pexman, Aguilera, et al., 2008; Siakaluk, Pexman, Sears, et al., 2008). Facilitation for high BOI words in lexical-semantic tasks is typically attributed to richer semantic representations of motoric interactions for those items (Pexman, 2012; Yap, Pexman, Wellsby, Hargreaves, \& Huff, 2012).

The facilitation of lexical-semantic processing for words with high BOI ratings versus words with low BOI ratings (hereafter the "BOI effect") is now well established, having been replicated and demonstrated across a variety of contexts. For example, the BOI effect has been observed in lexical 
decision tasks (LDT) (Siakaluk, Pexman, Aguilera, et al., 2008; Tillotson, Siakaluk, \& Pexman, 2008; Van Havermaet \& Wurm, 2014), semantic decision tasks (e.g., "Is it concrete?," "Is it easily imageable?"; Bennett, Burnett, Siakaluk, \& Pexman, 2011; Hansen, Siakaluk, \& Pexman, 2012; Hargreaves, Leonard, et al., 2012; Siakaluk, Pexman, Sears, et al., 2008; Tousignant \& Pexman, 2012; Yap et al., 2012), a semantic lexical decision task (i.e., "Is it a word?," then "Is it easily imageable?"; Siakaluk, Pexman, Sears, et al., 2008), sentence reading (Phillips, Sears, \& Pexman, 2012), and with child participants in auditory-word naming (6- to 7-year-old children; Inkster, Wellsby, Lloyd, \& Pexman, 2016) and in printed-word naming (8- to 9-year-old children; Wellsby \& Pexman, 2014). The BOI effect has also been observed using fMRI: High-BOI words were associated with higher levels of activation in the left inferior parietal lobule (a sensory association area involved in the planning of object-directed hand movements; van Elk, 2014) than were low-BOI words (Hargreaves, Leonard, et al., 2012). The fact that BOI effects are observed in lexical-semantic tasks suggests that children and adults routinely access information about their past sensorimotor experience with words' referents when making decisions about word meaning. Thus, BOI effects have been taken as evidence that sensorimotor information is important to representations of word meaning, although they cannot adjudicate between models that assume sensorimotor information is necessary for meaning activation (e.g., Glenberg, 2015) and those that assume sensorimotor information is simply activated as a by-product of meaning activation (e.g., Mahon, 2015).

The BOI effect has been frequently observed, but the precise nature of the information captured by the BOI dimension is not well specified. That is, $\mathrm{BOI}$ is a rather coarse semantic dimension that seemingly captures a variety of types of sensorimotor information and does not specify the nature of the interaction. Of particular relevance to the present study is the fact that the contribution of specific kinds of motor experience to facilitatory BOI effects has not previously been examined. The imprecision of the BOI measure makes it challenging to draw theoretical conclusions, because it is not clear what aspects of bodily information are being activated when word meanings are retrieved. The goal of the present study was to identify which aspects of motor experience drive BOI effects in order to better understand the BOI effects observed thus far, and to help refine theories of semantic representation by specifying the types of motor information involved in lexical-semantic processing.

\section{Candidate motor dimensions}

Several more specific motor dimensions may drive the BOI effects observed in lexical-semantic processing. In particular, there are specific aspects of object manipulation that have primarily been explored in the cognitive neuroscience, neuropsychology, and/or object recognition literatures, in which the stimuli are line drawings or photographs (e.g., Boronat et al., 2005; Garcea \& Mahon, 2012; Guérard, Lagacé, \& Brodeur, 2015; Madan, Chen, \& Singhal, 2016; Madan, Ng, \& Singhal, 2018; Salmon, Matheson, \& McMullen, 2014; Salmon, McMullen, \& Filliter, 2010; Tobia \& Madan, 2017). In the present work we considered whether these specific dimensions are related to BOI and whether they also explain word recognition behavior. We chose three candidate motor dimensions that have been shown in the object recognition literature to be related but also somewhat distinct aspects of objects' motor attributes (Guérard et al., 2015).

Graspability Graspability ratings provide a measure of how easily a person can grasp an object with one hand (Salmon et al., 2010). Many of the words that are rated as being high in BOI refer to objects that seem easily graspable (e.g., scissors). Thus, it is plausible that the facilitation effect observed for high-BOI words in lexical-semantic tasks is actually driven by sensorimotor information captured by graspability. Whereas graspability is based on how easily the hand can interact with a word's referent, BOI measures the ease with which any part of the body can interact with the word's referent. Nonetheless, the fact that the hands are primary for most human interactions with objects means that there is likely a good deal of overlap in the two dimensions. Effects of graspability have been investigated in both object recognition (Grèzes \& Decety, 2002; Guérard et al., 2015; Salmon et al., 2014) and word recognition (Amsel, Urbach, \& Kutas, 2012; Díez-Álamo, Díez, Alonso, Vargas, \& Fernandez, 2017) research. Two previous studies examined the relationship between graspability and BOI for word stimuli: Amsel et al. (2012) found a moderate correlation of $r=.62$ between the two dimensions for a set of 266 words, Díez-Álamo et al. found $r=.75$ for the same relationship in a set of 342 Spanish words. Thus, some evidence suggests that there is indeed overlap in the types of motoric information captured by these two measures, but neither previous study explored their relative contributions to lexical processes.

Ease of pantomime Ease of pantomime refers to how easily one can pantomime an object's functional use so that another individual could guess the identity of the object (Brodeur, Dionne-Dostie, Montreuil, \& Lepage, 2010; Guérard et al., 2015; Magnié, Besson, Poncet, \& Dolisi, 2003). For example, the referent for wharf would be difficult to pantomime, whereas doorknob would be easy (e.g., making a cupping shape with your hand and twisting at the wrist). This dimension depends on functional actions and so it likely taps how readily one can retrieve the conceptual knowledge associated with the object. Indeed, ease of pantomime is related to object naming latencies, with faster latencies for objects that are easier to 
pantomime (Guérard et al., 2015). Because BOI captures the ease with which the human body can interact with a word's referent, which typically occurs through functional use of the referent, it is plausible that ease of pantomime may be related to BOI for word stimuli and may drive or help drive the BOI effect.

Number of actions The number-of-actions dimension captures the number of functional actions that can typically be performed with an object (Guérard et al., 2015; Lagacé, Downing-Doucet, \& Guérard, 2013). This dimension also has a relationship with object-naming latencies, such that objects that afford more actions are associated with faster object naming (Guérard et al., 2015). Interaction between a word's referent and the human body typically occurs while using the referent for a functional purpose. For example, a word with a referent that affords few actions (e.g., fleck) is unlikely to be easy for the human body to interact with, whereas a word with a referent that affords many actions (e.g., baby: holding, cuddling, playing, etc.) is very easy for the human body to interact with. Thus, high-BOI words may afford relatively more actions and motor information regarding the number of actions a word's referent affords may drive or help drive the BOI effect.

\section{Other non-motor dimensions related to BOI}

Several other semantic dimensions might be related to BOI. In particular, we expected that animacy, size, danger, and usefulness might be relevant. BOI attempts to capture the ease with which the human body can interact with a word's referent and so it is possible that words with referents that are small, inanimate, nondangerous, and useful would be rated high on this dimension and that these factors may be related to the facilitation effect observed for BOI in lexical-semantic processing. Previous research suggests that each of these four dimensions may be related to semantic processing. For instance, there is evidence of faster recognition of animate than of inanimate objects (Proverbio, Del Zotto, \& Zani, 2007) and of better memory for words referring to animate concepts than for words referring to inanimate concepts (e.g., Bonin, Gelin, \& Bugaiska, 2014). There is also evidence that words referring to large objects are recognized faster than words referring to small objects (Sereno, O’Donnell, \& Sereno, 2009), but at the same time, there is evidence that object size does not affect word recognition (Kang, Yap, Tse, \& Kurby, 2011). Effects of danger, usefulness, and their interactions with BOI have been characterized in recent studies by Wurm and colleagues (e.g., Van Havermaet \& Wurm, 2014, 2017; Wurm, 2007; Wurm \& Seaman, 2008). Although some dangerous objects are frequent targets of interaction, it seemed possible that the caution required when interacting with such objects might lead to lower BOI values since those assess relative "ease" of interaction. We included each of these variables in the present study in order to assess their relationships with BOI, and to allow us to examine effects of the candidate motor dimensions independent of these other nonmotor factors.

\section{Present study}

Here we investigated several semantic dimensions that could be related to BOI effects observed in lexical-semantic processing, focusing in particular on the motor dimensions described by Guérard et al. (2015) as influential for object recognition. We collected new ratings so that we could examine effects for word stimuli. Our stimuli were 621 words that had been previously rated for BOI (Bennett et al., 2011; Tillotson et al., 2008). We used hierarchical regression analyses to examine relationships between our candidate dimensions and BOI ratings. We then examined whether our candidate dimensions predicted variance in semantic decision behavior using response data from the Calgary Semantic Decision (Calgary SDT) Project (Pexman, Heard, Lloyd, \& Yap, 2017). In the Calgary SDT Project, the decision category was "is it concrete or abstract?." We chose the behavioral data from this task to examine effects of the candidate motor dimensions because in this task a relatively large amount of variance is explained by semantic variables (Pexman et al., 2017). Less variance is typically explained by semantic variables in the lexical decision task (LDT; see Pexman, 2012, for a review), but because the LDT is so widely used, we also examined whether our candidate dimensions predicted variance in LDT behavior using response data from the English Lexicon Project (Balota et al., 2007). We predicted that the semantic variables would account for less variance in LDT than SDT.

If a single motor dimension underlies the BOI dimension, then a significant amount of unique variance in BOI ratings should be predicted by ratings on that motor dimension. If multiple motor dimensions underlie the BOI dimension, then ratings on several motor dimensions should significantly predict variance in BOI ratings. Finally, the motor dimension(s) found to predict unique variance in $\mathrm{BOI}$ ratings will likely also predict SDT and LDT response behavior, and may be a stronger predictor of SDT and/or LDT behavior than is BOI.

\section{Method}

\section{Participants}

A total of 490 participants were recruited for the ratings task. All participants were undergraduate students at the University of Calgary who received partial course credit in exchange for participation. Each participant provided ratings on one of the seven ratings scales for half of the stimuli, with 70 participants 
assigned to each rating scale ( 35 to each half of the stimuli). A total of 23 participants did not complete their assigned survey. In addition, the participants' data were removed before analyses if (1) they provided the same rating for more than seven words consecutively (graspability: one participant; ease of pantomime: no participants; number of actions: one; animacy: two; size: one; danger: four; usefulness: one) or (2) their overall mean rating was more than two standard deviations from the group mean (graspability: six participants; ease of pantomime: three; number of actions: four; animacy: three; size: three; danger: six; usefulness: one). The resulting group sizes for each semantic dimension (for each half of the stimuli, respectively; see below) were as follows: graspability (29 and 29), ease of pantomime (29 and 32), number of actions (28 and 33), animacy (31 and 29), size (31 and 34), danger (29 and 32 ), and usefulness (35 and 30).

\section{Stimuli}

The stimuli were 621 words from the Tillotson et al. (2008) and Bennett et al. (2011) BOI norms that were also included as stimuli in the Calgary SDT (Pexman et al., 2017) and English Lexicon (Balota et al., 2007) Projects. Stimuli were divided into two lists consisting of 311 and 310 words, such that the two lists were matched for mean BOI rating (3.57 and 3.58, respectively). Descriptive characteristics for these stimuli can be found in Table 1.

Table 1. Mean descriptive statistics (standard deviations in parentheses) for the 618 stimuli included in the body-object interaction (BOI) and lexical decision task (LDT) analyses and the 521 stimuli included in the semantic decision task (SDT) analyses

\begin{tabular}{lll}
\hline Variable & $\begin{array}{l}\text { Mean } \\
(n=618)\end{array}$ & $\begin{array}{l}\text { Mean } \\
(n=521)\end{array}$ \\
\hline Length & $6.02(1.92)$ & $6.31(1.93)$ \\
Log frequency & $2.31(0.75)$ & $2.29(0.75)$ \\
Graspability & $3.39(1.48)$ & $4.22(1.41)$ \\
Ease of pantomime & $3.84(1.10)$ & $4.03(1.06)$ \\
Number of actions & $2.12(0.61)$ & $2.21(0.59)$ \\
Animacy & $2.73(1.32)$ & $2.78(1.42)$ \\
Size & $3.19(1.05)$ & $3.08(1.06)$ \\
Log danger & $0.25(0.16)$ & $0.25(0.16)$ \\
Usefulness & $3.18(0.70)$ & $3.21(0.70)$ \\
Age of acquisition & $7.62(2.47)$ & $7.26(2.34)$ \\
BOI & $3.57(1.39)$ & $3.93(1.21)$ \\
SDT latency (ms) & - & $872.40(113.37)$ \\
SDT accuracy $(\%)$ & - & $92.51(10.16)$ \\
LDT latency $(\mathrm{ms})$ & $691.85(86.63)$ & - \\
LDT accuracy $(\%)$ & $91.20(12.11)$ & - \\
\hline
\end{tabular}

\section{Procedure}

The two stimulus lists were used to make two versions of an online questionnaire for ratings on each of the seven semantic dimensions; these 14 online questionnaires were created using Qualtrics. On each ratings questionnaire, words were presented in random order, 30 to a screen, with the relevant rating scale presented below each word. The ratings instructions are described in brief here and full versions of the ratings instructions for each semantic dimension are available in the Appendix. For ratings of graspability, ease of pantomime, and number of actions, scale anchors were adapted from those used by Guérard et al. (2015). For ratings of danger and usefulness, the scale anchors were adapted from those used by Wurm (2007).

Graspability Participants were asked to rate how easily each word's referent could be grasped using one hand from 1 to 7 (1 = cannot be grasped, 7 = very graspable .

Ease of pantomime Participants were asked to rate how easily each word's referent can be pantomimed from 1 to 7 ( $1=$ difficult, 7 = easy).

Number of actions Participants were asked to rate the number of actions that can typically be performed with a word's referent from 0 (no actions) to $6+$ (more than six actions).

Animacy Participants were asked to rate how alive and capable of self-propelled motion each word's referent is from 1 to 7 (1 = inanimate, 7 = animate).

Size Participants were asked to rate the size of a word's referent from 1 to 7 ( 1 = very small, $7=$ very large $)$.

Danger Participants were asked to rate how dangerous a word's referent is for human survival from 1 to 7 ( $1=$ not at all dangerous, 7 = extremely dangerous).

Usefulness Participants were asked to rate how useful a word's referent is for human survival from 1 to 7 (1=not at all useful, 7 = extremely useful).

\section{Results}

The mean ratings and standard deviations for all 621 words for each of the seven semantic rating dimensions are available at https://osf.io/4jrcf/. The distributions for the seven semantic rating dimensions are presented in Fig. 1. The semantic variable danger showed positive skew (1.97) and so was $\log _{10}$-transformed. 
Graspability

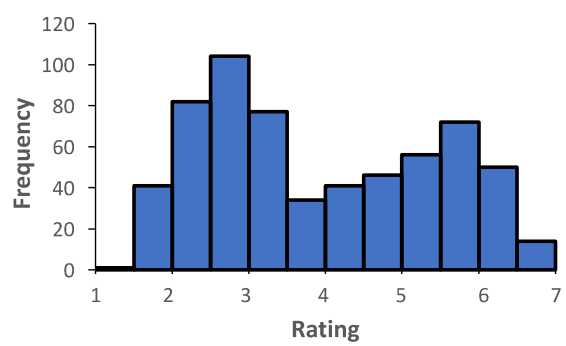

Ease of Pantomime

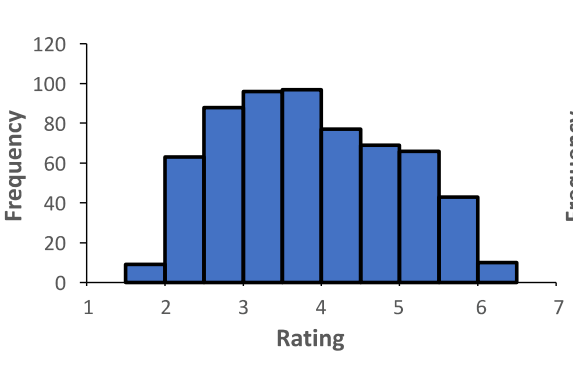

Number of Actions

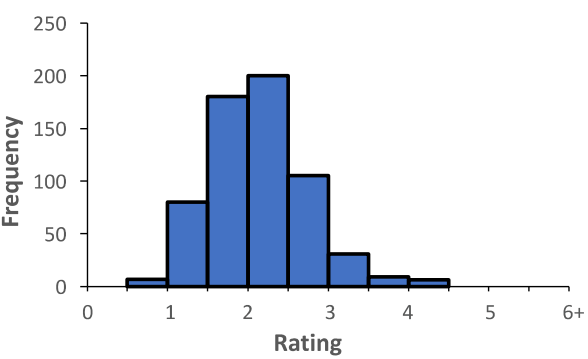

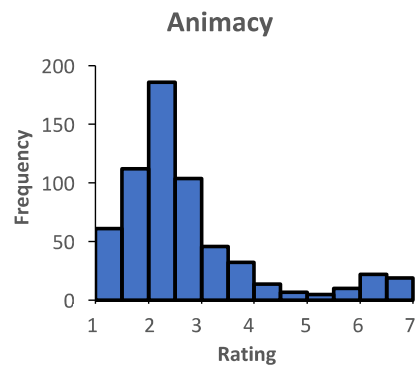

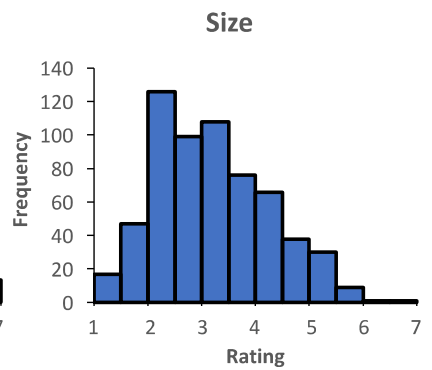

Fig. 1. Distributions of semantic variable ratings. Histograms for each semantic variable are depicted. The $x$-axes depict the full range of each rating scale (1-7; except for number of actions, $0-6+$ ), and the $y$-axes

To explore the relationships between the seven semantic rating dimensions, BOI, and SDT and LDT performance, we conducted six main analyses. In the first regression analysis we examined the extent to which the semantic dimensions predicted BOI ratings, after controlling for lexical factors that could also influence BOI ratings. In the second and third analyses, we examined the extent to which the semantic dimensions predicted SDT response latencies and SDT response accuracy, again after controlling for lexical factors that usually influence SDT performance. In the fourth and fifth analyses, we examined the extent to which semantic dimensions predicted LDT response latencies and LDT response accuracy, again after controlling for lexical factors that usually influence LDT performance. Finally, we conducted analyses to examine whether BOI or the three candidate motor dimensions were better predictors of lexical-semantic processing.

The variables used in the analyses were divided into two clusters: lexical control variables and semantic dimensions. The lexical control variables were those that have been shown to be the most important nonsemantic predictors of lexical and semantic decision performance: word length (Balota et al., 2007) and frequency (log-transformed SUBTLEX word frequency; Brysbaert \& New, 2009). The semantic dimensions included BOI (Bennett et al., 2011; Tillotson et al., 2008) and our collected ratings: graspability, ease of pantomime, number of actions (Guérard et al., 2015), animacy (Amsel et al., 2012), size, danger and usefulness (Wurm, 2007), and age of acquisition (AOA; Kuperman, Stadthagen-Gonzalez, \& Brysbaert, 2012). We included
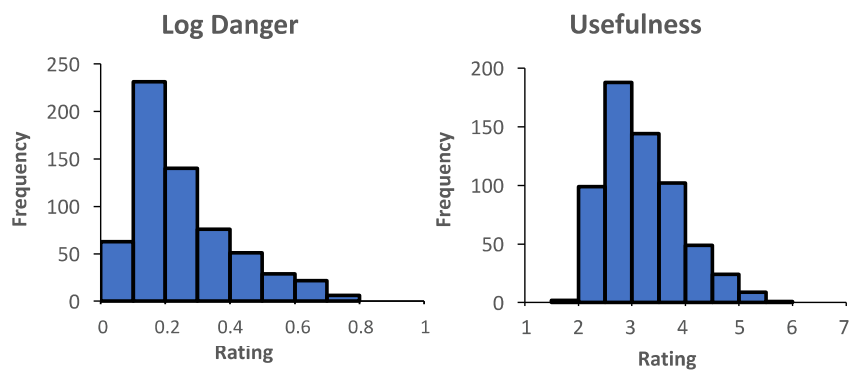

depict the frequency of items within each rating bin. Bins were created for each 0.5 increment of the rating scale

AOA because this variable has previously been shown to be related to BOI (Connell \& Lynott, 2016). That is, high-BOI words tend to be acquired earlier than low-BOI words (Thill \& Twomey, 2016). Descriptive characteristics for each of these variables are presented in Table 1. AOA ratings (Kuperman et al., 2012) were not available for two words, and lexical decision data were not available for one word, so we used a final set of 618 words for the analyses of BOI ratings and LDT response data. For analyses of the SDT response data, we further restricted the item list to the 521 words that received "concrete" responses in the Calgary SDT Project data. We did not include in our analyses the 97 words that received "abstract" responses in that dataset because past research has shown that concrete and abstract words have some distinct relationships with semantic dimensions (Pexman et al., 2017; Pexman \& Yap, 2018).

Correlations between all of the variables are presented in Table 2. The semantic dimension most highly correlated with BOI ratings was graspability. In addition, the correlation between graspability and size was relatively high, with smaller objects being rated as easier to grasp. The relationships between the other semantic dimensions were modest, suggesting that each dimension captures slightly different aspects of meaning.

\section{Hierarchical regression analyses}

Regression 1: BOI ratings The control variables were entered in Step 1 and the semantic dimensions were entered in Step 2 of a hierarchical regression with BOI ratings as the 
dependent variable. The results of the regression analysis are presented in Table 3. Both length and frequency were significant predictors of BOI ratings, with higher BOI ratings for longer, more frequent words. Together, the semantic dimensions accounted for a significant amount of variance in BOI ratings. Significant, unique relationships were observed for graspability, ease of pantomime, number of actions, and animacy. That is, BOI ratings were higher for words with referents that were easier to grasp, were easier to pantomime, afforded more actions, and were less animate. The relationships between BOI and AOA, danger, and usefulness were not significant.

Regression 2: SDT response latencies The lexical control variables were entered in Step 1 and the semantic dimensions in Step 2 of a hierarchical regression analysis with SDT response latencies as the dependent variable. The regression results are presented in Table 4. Both length and frequency were significant predictors of SDT response latencies, with faster latencies for longer, more frequent words. The semantic dimensions predicted a significant amount of additional variance. Significant relationships were observed for graspability, ease of pantomime, number of actions, animacy, size, AOA, and BOI. More specifically, SDT latencies were faster for words with referents that were easier to grasp, were easier to pantomime, afforded more actions, were more animate, were larger in size, were acquired earlier in life, and were higher in BOI. The relationships between SDT latencies and danger and usefulness were not significant.

Regression 3: SDT accuracy The control variables were entered in Step 1 and the semantic dimensions in Step 2 of a hierarchical regression analysis with SDT response accuracies as the dependent variable. The regression results are presented in Table 5. Both length and frequency were significant predictors of SDT response accuracy, with greater accuracy for longer, more frequent words. The semantic dimensions predicted a significant amount of additional variance. Significant relationships were observed for graspability, ease of pantomime, number of actions, animacy, size, AOA, and BOI. That is, SDT responses were more accurate for words with referents that were easier to grasp, were easier to pantomime, afforded more actions, were more animate, were larger in size, were acquired earlier in life, and had higher BOI. The relationships between SDT accuracy and danger and usefulness were not significant.

For both SDT response latencies and accuracy, parallel analyses were also conducted on the trial-level data using a linear mixed-effects approach. These analyses yielded results comparable to those from our hierarchical regressions. That is, the same significant relationships observed 
Table 3. Regression coefficients from item-level regression analyses for body-object interaction ratings

\begin{tabular}{llllll}
\hline Variable & $B$ & $S E B$ & $\beta$ & $R^{2}$ & $\Delta R^{2}$ \\
\hline Step 1 (Control variables) & & & & $.16^{* * *}$ & $.16^{* * * *}$ \\
$\quad$ Length & 0.27 & 0.03 & $.37^{* * * *}$ & & \\
$\quad$ Log frequency & 0.42 & 0.07 & $.23^{* * *}$ & & \\
Step 2 (Semantic variables) & & & & $.69^{* * *}$ & $.53^{* * *}$ \\
$\quad$ Graspability & 0.48 & 0.04 & $.51^{* * *}$ & & \\
Ease of pantomime & 0.24 & 0.04 & $.19^{* * *}$ & & \\
Number of actions & 0.64 & 0.08 & $.28^{* * *}$ & & \\
Animacy & -0.17 & 0.03 & $-.16^{* * *}$ & & \\
Size & -0.08 & 0.05 & -.06 & & \\
Log danger & -0.23 & 0.27 & -.03 & & \\
Usefulness & -0.04 & 0.06 & -.02 & & \\
AOA & -0.01 & 0.02 & -.03 & & \\
**** $p<.001$. & & & & & \\
$p<.03$ & & & &
\end{tabular}

in the hierarchical regression analyses were also observed using linear mixed-effects analyses.

Regression 4: LDT response latencies The lexical control variables were entered in Step 1 and the semantic dimensions in Step 2 of a hierarchical regression analysis with LDT response latencies as the dependent variable. The regression results are presented in Table 6. Both length and frequency were significant predictors of LDT response latencies, with faster latencies for shorter, more frequent words. The semantic dimensions predicted a significant amount of additional variance, although, as expected,

Table 4. Regression coefficients from item-level regression analyses for semantic decision task latencies

\begin{tabular}{llllll}
\hline Variable & $B$ & $S E B$ & $\beta$ & $R^{2}$ & $\Delta R^{2}$ \\
\hline Step 1 (Control variables) & & & & $.16^{* * *}$ & $.16^{* * * *}$ \\
$\quad$ Length & -9.43 & 2.40 & $-.16^{* * *}$ & & \\
Log frequency & -60.48 & 6.17 & $-.40^{* * *}$ & & \\
Step 2 (Semantic variables) & & & & $.47^{* * *}$ & $.31^{* * * *}$ \\
Graspability & -23.50 & 5.13 & $-.29^{* * *}$ & & \\
Ease of pantomime & -10.88 & 4.94 & $-.10^{*}$ & & \\
Number of actions & -25.23 & 9.61 & $-.13^{* *}$ & & \\
Animacy & -11.39 & 2.88 & $-.14^{* * *}$ & & \\
Size & -19.35 & 5.98 & $-.18^{* *}$ & & \\
Log danger & -21.43 & 32.52 & -.03 & & \\
Usefulness & -0.74 & 7.49 & -.01 & & \\
AOA & 16.42 & 2.23 & $.34^{* * * *}$ & \\
BOI & -10.48 & 4.84 & $-.11^{* *}$ & \\
\hline
\end{tabular}

${ }^{*} p<.05,{ }^{* *} p<.01,{ }^{* * *} p<.001$.
Table 5. Regression coefficients from item-level regression analyses for semantic decision task accuracy

\begin{tabular}{llllll}
\hline Variable & $B$ & $S E B$ & $\beta$ & $R^{2}$ & $\Delta R^{2}$ \\
\hline Step 1 (Control variables) & & & & $.14^{* * *}$ & $.14^{* * * *}$ \\
$\quad$ Length & 0.02 & 0.00 & $.31^{* * *}$ & & \\
$\quad$ Log frequency & 0.04 & 0.01 & $.28^{* * *}$ & & \\
Step 2 (Semantic variables) & & & & $.42^{* * *}$ & $.28^{* * *}$ \\
$\quad$ Graspability & 0.01 & 0.01 & $.17^{* *}$ & & \\
Ease of pantomime & 0.01 & 0.01 & $.14^{* *}$ & & \\
Number of actions & 0.03 & 0.01 & $.18^{* *}$ & & \\
Animacy & 0.01 & 0.00 & $.11^{* *}$ & & \\
Size & 0.01 & 0.01 & $.15^{* *}$ & & \\
Log danger & 0.02 & 0.03 & .03 & & \\
Usefulness & 0.00 & 0.01 & .01 & & \\
AOA & -0.01 & 0.00 & $-.23^{* * *}$ & & \\
BOI & 0.02 & 0.01 & $.24^{* * *}$ & & \\
${ }^{*} p<.05,{ }^{* *} p<.01,{ }^{* * *} p<.001$. & & & &
\end{tabular}

much less than they explained in the analysis of SDT latencies. A significant relationship was observed only for AOA, in that LDT latencies were faster for words that were acquired earlier in life. The relationships between LDT latencies and graspability, ease of pantomime, number of actions, animacy, size, danger, usefulness, and BOI were not significant.

Regression 5: LDT accuracy The control variables were entered in Step 1 and the seman1tic dimensions in Step 2 of a hierarchical regression analysis with LDT response

Table 6. Regression coefficients from item-level regression analyses for lexical decision task latencies

\begin{tabular}{llllll}
\hline Variable & $B$ & SEB & $\beta$ & $R^{2}$ & $\Delta R^{2}$ \\
\hline Step 1 (Control variables) & & & & $.41^{* * *}$ & $.41^{* * * *}$ \\
$\quad$ Length & 9.42 & 1.42 & $.21^{* * * *}$ & & \\
$\quad$ Log frequency & -66.04 & 3.62 & $-.57^{* * *}$ & & \\
Step 2 (Semantic variables) & & & & $.45^{* * *}$ & $.04^{* * * *}$ \\
Graspability & -0.23 & 3.72 & -.00 & & \\
Ease of pantomime & -3.83 & 3.61 & -.05 & & \\
Number of actions & -8.00 & 6.84 & -.06 & & \\
Animacy & 1.82 & 2.18 & .03 & & \\
Size & -2.28 & 4.30 & -.03 & & \\
Log danger & 7.30 & 22.58 & .01 & & \\
Usefulness & -8.50 & 5.30 & -.07 & & \\
AOA & 5.67 & 1.60 & $.16^{* * * *}$ & & \\
BOI & 0.13 & 3.38 & .00 & & \\
\hline
\end{tabular}

**** $p<.001$. 
Table 7. Regression coefficients from item-level regression analyses for lexical decision task accuracy

\begin{tabular}{llllll}
\hline Variable & $B$ & $S E B$ & $\beta$ & $R^{2}$ & $\Delta R^{2}$ \\
\hline Step 1 (Control variables) & & & & $.32^{* * *}$ & $.32^{* * * *}$ \\
$\quad$ Length & 0.02 & 0.00 & $.27^{* * * *}$ & & \\
$\quad$ Log frequency & 0.09 & 0.01 & $.54^{* * *}$ & & \\
Step 2 (Semantic variables) & & & & $.42^{* * *}$ & $.10^{* * * *}$ \\
$\quad$ Graspability & 0.02 & 0.01 & $.23^{* * *}$ & & \\
Ease of pantomime & 0.01 & 0.01 & .04 & & \\
Number of actions & 0.02 & 0.01 & .10 & & \\
Animacy & -0.00 & 0.00 & -.03 & & \\
Size & 0.02 & 0.01 & $.18^{* * *}$ & & \\
Log danger & 0.02 & 0.03 & .03 & & \\
Usefulness & -0.00 & 0.01 & -.02 & & \\
AOA & -0.01 & 0.00 & $-.28^{* * *}$ & & \\
BOI & -0.01 & 0.01 & -.08 & & \\
\hline *** $p<.01,{ }^{* * * *} p<.001$. & & & & & \\
$p$ & & & &
\end{tabular}

accuracies as the dependent variable. The regression results are presented in Table 7. Both length and frequency were significant predictors of LDT response accuracy, with greater accuracy for longer, more frequent words. The semantic dimensions predicted a significant amount of additional variance. Significant relationships were observed for graspability, size, and AOA. That is, LDT responses were more accurate for words with referents that were easier to grasp, larger, and acquired earlier in life. The relationships between LDT accuracy and ease of pantomime, number of actions, animacy, danger, usefulness, and BOI were not significant.

Regression 6: Comparative analyses We conducted one final set of analyses to compare the amounts of variance in SDT and LDT behavioral performance explained by BOI with that explained by our three candidate motor dimensions (graspability, ease of pantomime, and number of actions). Again, we entered the lexical factors at Step 1 of these analyses, and then entered BOI or the three candidate motor dimensions at Step 2 of the analyses, along with AOA. We also calculated BIC values (Wagenmakers, 2007) in order to compare the effectiveness of the models containing the two different sets of predictors, for each dependent variable. The results of these analyses are presented in Table 8, with results presented separately for analyses of SDT latencies, SDT accuracy, LDT latencies, and LDT accuracy. BIC analyses show that for SDT latencies and accuracy, the models with the candidate motor dimensions together were more effective than the models with BOI. In contrast, for LDT latencies and accuracy, the models with
BOI were more effective than those with the candidate motor dimensions.

\section{Discussion}

The goal of the present study was to investigate which aspects of motor experience drive BOI effects in lexical-semantic processing. To do so, we collected ratings for 621 words on seven semantic dimensions, including three motor attributes (graspability, ease of pantomime, number of actions) and four other nonmotor dimensions that we thought might be related to BOI (animacy, size, danger, and usefulness). We found that the semantic dimension ratings collected in the present study could explain a considerable amount $(53 \%)$ of the variance in the BOI ratings that had been collected in previous studies (Bennett et al., 2011; Tillotson et al., 2008). Thus, the present findings offer some insights about factors that are related to BOI ratings. Graspability was the most significant predictor of BOI ratings, with high-BOI concepts tending to be easier to grasp. This is presumably because upper-limb interactions are important to human motor behavior and thus to the relative ease of our interactions with objects. Whereas graspability is based only on interactions of the hands with objects, BOI may also include interactions with other body parts. For instance, interactions of the feet with objects are likely important, as indicated by the moderately high BOI ratings for foot-related words like stair (5.23) and step (4.6). In addition, it seems likely that a more general bodily sense like that described by Borghi and Cimatti (2010) is also relevant. That is, a proprioceptive sense, involving an awareness of the body in place and time, may be part of what participants consider when they provide ratings of BOI. This might explain why words like fire and shower, which afford few opportunities for grasping, nonetheless receive moderate BOI ratings (4.27 and 4.33).

The seven semantic variables together explained a substantial amount of variance in SDT responses and, as expected, much less variance in LDT responses. SDT responses rely heavily on semantic processing, whereas LDT responses rely more heavily on lexical and orthographic processing (e.g., Pexman \& Yap, 2018; Taikh, Hargreaves, Yap, \& Pexman, 2014; Yap et al., 2012). Indeed, the lexical control variables (length and frequency) explained considerably more variance in LDT performance than in SDT performance. Of the seven semantic variables, graspability was one of the two most consistent semantic predictors of lexical-semantic processing, in that high graspability was associated with faster and more accurate SDT responses and with more accurate LDT responses. The other consistent semantic predictor was size, but in a way that suggests that it works in opposition to BOI. The zero-order correlations (Table 2) show that, as 
Table 8. Regression coefficients from item-level regression analyses comparing the variance explained by body-object interaction (BOI) versus motor dimensions

\begin{tabular}{|c|c|c|c|c|c|c|c|c|c|c|c|c|c|}
\hline \multirow[b]{2}{*}{ Variable } & \multicolumn{6}{|l|}{ BOI } & \multicolumn{6}{|c|}{ Motor Dimensions } & \multirow[b]{2}{*}{$\Delta \mathrm{BIC}$} \\
\hline & $B$ & $S E B$ & $\beta$ & $s r$ & $R^{2}$ & $\Delta R^{2}$ & $B$ & $S E B$ & $\beta$ & $s r$ & $R^{2}$ & $\Delta R^{2}$ & \\
\hline SDT Latencies & & & & & $.40^{* * * *}$ & $.24^{* * * *}$ & & & & & $.44^{* * * *}$ & $.28^{* * * *}$ & -25.41 \\
\hline BOI & -19.61 & 3.53 & $-.21^{* * *}$ & -.19 & & & & & & & & & \\
\hline Graspability & & & & & & & -14.91 & 2.98 & $-.19^{* * * *}$ & -.17 & & & \\
\hline Ease of pantomime & & & & & & & -16.20 & 4.61 & $-.15^{* * * *}$ & -.12 & & & \\
\hline Number of actions & & & & & & & -38.07 & 9.08 & $-.20^{* * * *}$ & -.14 & & & \\
\hline $\mathrm{AOA}$ & 22.53 & 2.02 & $.46^{* * * *}$ & .38 & & & 17.04 & 2.17 & $.35^{* * *}$ & .26 & & & \\
\hline $\begin{array}{l}\text { SDT Accuracy } \\
\text { BOI }\end{array}$ & 0.03 & 0.00 & $.30^{* * * *}$ & .28 & $.36^{* * *}$ & $.21^{* * * *}$ & & & & & $.38^{* * * *}$ & $.24^{* * * *}$ & -12.14 \\
\hline Graspability & & & & & & & 0.01 & 0.00 & $.18^{* * * *}$ & .16 & & & \\
\hline Ease of pantomime & & & & & & & 0.02 & 0.00 & $.21^{* * * *}$ & .16 & & & \\
\hline Number of actions & & & & & & & 0.05 & 0.01 & $.26^{* * * *}$ & .18 & & & \\
\hline $\mathrm{AOA}$ & -0.02 & 0.00 & $-.35^{* * *}$ & -.29 & & & -0.01 & 0.00 & $-.23^{* * * *}$ & -.17 & & & \\
\hline $\begin{array}{l}\text { LDT Latencies } \\
\text { BOI }\end{array}$ & -4.73 & 2.62 & -.07 & -.06 & $.45^{* * *}$ & $.04^{* * * *}$ & & & & & $.46^{* * * *}$ & $.04^{* * * *}$ & 8.38 \\
\hline Graspability & & & & & & & -0.46 & 2.28 & -.01 & -.01 & & & \\
\hline Ease of pantomime & & & & & & & -6.32 & 3.53 & -.08 & -.06 & & & \\
\hline Number of actions & & & & & & & -12.96 & 6.95 & -.09 & -.06 & & & \\
\hline $\mathrm{AOA}$ & 7.77 & 1.50 & $.21^{* * * *}$ & .17 & & & 6.50 & 1.66 & $.17^{* * * *}$ & .13 & & & \\
\hline LDT Accuracy & & & & & $.40^{* * * *}$ & $.09^{* * * *}$ & & & & & $.40^{* * * *}$ & $.09^{* * * *}$ & 5.23 \\
\hline BOI & 0.01 & 0.00 & .06 & .05 & & & & & & & & & \\
\hline Graspability & & & & & & & 0.00 & 0.00 & .04 & .04 & & & \\
\hline Ease of pantomime & & & & & & & 0.01 & 0.01 & .06 & .05 & & & \\
\hline Number of actions & & & & & & & 0.02 & 0.01 & $.11^{*}$ & .08 & & & \\
\hline $\mathrm{AOA}$ & -0.02 & 0.00 & $-.32^{* * *}$ & -.26 & & & -0.01 & 0.00 & $-.28^{* * *}$ & -.20 & & & \\
\hline
\end{tabular}

$\triangle \mathrm{BIC}$ was calculated using Wagenmakers's (2007) method for comparing nonnested models. Model 1 = BOI; Model $2=$ graspability, ease of pantomime, and number of actions. Negative BIC values indicate a higher BIC for Model 2, indicating that it is preferred over Model 1. Positive values indicate a higher BIC for Model 1, indicating that it is preferred over Model 2. ${ }^{*} p<.05,{ }^{* * * *} p<.001$.

expected, size was negatively related to BOI; that is, words that refer to small objects tend to have higher BOI ratings. The analyses of lexical-semantic processing, however, showed that words that refer to large objects have faster and more accurate SDT responses and more accurate LDT responses. These facilitatory effects of referent object size are consistent with those reported by Sereno et al. (2009; cf. Kang et al., 2011). Sereno et al. attributed their size effects to faster retrieval of visual representations for large than for small objects, and they took such effects as evidence that sensorimotor information is automatically accessed when reading a word.

Similarly, the patterns observed for the animacy variable suggest that its effects also work in opposition to those of BOI. As expected, animacy was negatively related to BOI ratings, such that more animate objects tended to have lower BOI ratings; many animate objects (e.g., tiger, rhinoceros) do not afford easy interaction. In lexical-semantic processing, however, animacy facilitated responses: in SDT, responses were faster and more accurate for words referring to more animate objects. The present finding of an animacy effect in SDT extends the effects observed previously in other contexts, such as object recognition (Proverbio et al., 2007), and memory (e.g., Bonin et al., 2014). Animacy effects have been attributed to the survival advantage for these concepts (e.g., Nairne, 2010), in that animate objects play an important role in human survival, as both predators and prey. Thus, although size and animacy are related to BOI ratings, they are likely not drivers of the facilitatory BOI effect observed in lexical-semantic processing.

Instead, our results suggest that, from among the variables we measured in the present study, the three motor dimensions are the most likely drivers of BOI effects in lexical-semantic processing. Furthermore, the comparative analyses showed that these three variables are more effective than BOI at explaining variance in SDT, and somewhat less effective at 
explaining variance in LDT responses. We speculate that the candidate motor dimensions are particularly effective predictors in SDT because the concrete/abstract decision depends on extensive semantic processing and a strategy of focusing on tactile and tangible features (Hargreaves, White, Pexman, Pittman, \& Goodyear, 2012). Specific dimensions of motor information better predict this more specific semantic processing in SDT. In contrast, in LDT the focus is largely on orthographic processing, and although semantic processing is relevant, it is less extensive and less specific. The single predictor of BOI is effective in that case.

Depending on their goals, researchers may want to use these more specific motor dimensions rather than BOI to examine whether particular aspects of motor information are accessed in language or cognitive tasks. For instance, in past research less-specific measures of motor-related attributes of functionality and manipulability have been examined and found to influence a variety of cognitive domains, including memory, attention, and semantic processing (e.g., Creem \& Proffitt, 2001; Hauk, Johnsrude, \& Pulvermüller, 2004; Just, Cherkassky, Aryal, \& Mitchell, 2010; Madan, 2014, 2017; Madan \& Singhal, 2012; Montefinese, Ambrosini, Fairfield, \& Mammarella, 2013; Shebani \& Pulvermüller, 2013). The ratings collected in the present study offer researchers the opportunity to investigate the contributions of motor information in a more fine-grained way, helping advance our understanding of the role that motor information plays in human cognition.

Author note This work was supported by the Natural Sciences and Engineering Research Council of Canada (NSERC), through a CREATE Scholarship to A.H., and Discovery Grants to A.B.P. and P.M.P.

\section{Appendix}

\section{Written instructions for the graspability rating task}

The referents that words refer to vary in terms of how graspable they are. Something that is graspable can be grasped using one hand whereas something that is not graspable cannot.

In this study your task is to rate each word's referent based on how graspable it is. Ratings will be made on a scale from 1 to 7. Any referent you believe not graspable should be rated as a 1 and any referent you believe to be very graspable should be rated a 7 .

For example, the word "beach" should be rated as a 1 since its referent cannot be grasped with one hand whereas the word "scissors" should be rated as a 7 since its referent can easily be grasped with one hand.
When rating a referent, try to be as accurate as possible, but do not spend too much time on it.

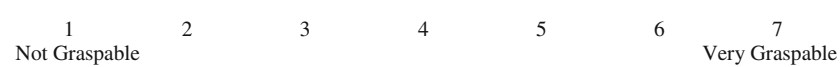

\section{Written instructions for the ease of pantomime rating task}

Objects differ in the extent to which a person can think of an action involving that object. For some objects it is easier to think of an action than for others. The purpose of the present experiment is to rate words regarding how easily a person could pantomime or act out the use of their referent (what the word is referring to).

In this case a pantomime is defined as a concept that can be expressed with a few simple gestures. For example, interactions with a "hammer" are probably quite easy to pantomime (e.g., a downward swinging gesture). It is more difficult to pantomime interactions with a "ceiling."

Any referent that is easy to pantomime should be given a high rating. Any referent that is difficult to pantomime should be given a low rating. Your ratings will be made on a 1 to 7 scale, a value of 1 indicates that you think the referent is very difficult to pantomime and a value of 7 indicating that the referent is very easy to pantomime. Values of 2 to 6 indicate intermediate ratings. Click on the most appropriate value for each referent. When rating a referent, try to be as accurate as possible, but do not spend too much time on it.

$\begin{array}{cccccccc}1 & 2 & 3 & 4 & 5 & 6 & \begin{array}{c}7 \\ \text { Very Easy } \\ \text { to Pantomime }\end{array} \\ \text { to Pantomime } & & & & & & \end{array}$

\section{Written instructions for the number of actions rating task}

Some objects can be used to perform multiple actions (e.g., a dog can be pet, brushed, hugged, etc.) while other objects can only be used for one particular action (e.g., chopsticks can be used for picking up food). For each word, your task is to estimate the number of actions that you can typically perform with the word's referent (what the word refers to). For each object, click on the value corresponding to the number of possible actions.

For example, "scissors" would probably have one action as it would typically be used to cut whereas "ball" would likely have multiple actions as it can be thrown, caught, kicked, etc.

*please note: although it may be possible to use the word's referent for other actions (e.g., using a screwdriver to hammer a nail) we ask that you rate the referent on the number of actions it would typically be used for. 
Your ratings will be made on a 0 to $6+$ scale. Any referent with 6 or more associated actions should be rated a $6+$. When rating a referent, try to be as accurate as possible, but do not spend too much time on it.

$\begin{array}{cccccccc}0 & 1 & 2 & 3 & 4 & 5 & 6+ \\ \text { No Action } & & & & & & & \text { Many }\end{array}$

\section{Written instructions for the animacy rating task}

The referents that words refer to vary in terms of whether they are animate or inanimate. An animate thing is something that is living and capable of self-propelled motion whereas an inanimate thing is something that is nonliving and incapable of self-propelled motion.

In this study your task is to rate the animacy of the word's referent. Ratings will be made on a scale from 1 to 7 . Any referent you believe is completely inanimate should be rated a 1 and any referent you believe to be completely animate should be rated a 7 .

For example, the word "wall" should be rated as a 1 since its referent is completely inanimate whereas the word "rabbit" should be rated as a 7 since its referent is completely animate.

When rating a referent, try to be as accurate as possible, but do not spend too much time on it.

$\begin{array}{ccccccc}1 & 2 & 3 & 4 & 5 & 6 & 7 \\ \text { Inanimate } & & & & & & \end{array}$

\section{Written instructions for the size rating task}

The referents that words refer to greatly vary in size. In this study your task is to rate the size of the word's referent from very small to very large. Ratings will be made on a scale from 1 to 7 . Any referent you believe is very small should be rated a 1 and any referent you believe to be very large should be rated a 7 .

For example, the word "pin" should be rated as a 1 since its referent is very small whereas the word "Earth" should be rated as a 7 since its referent is very large.

When rating a referent, try to be as accurate as possible, but do not spend too much time on it.

$\begin{array}{ccccccc}1 & 2 & 3 & 4 & 5 & 6 & 7 \\ \text { Small } & & & & & & \text { Large }\end{array}$

\section{Written instructions for the danger rating task}

We would like you to rate how dangerous for human survival you think each word's referent is.

Your ratings will be made on a 1 to 7 scale, a value of 1 indicating that the referent is not at all dangerous to human survival and a value of 7 indicating that the referent is extremely dangerous for human survival. Values of 2 to 6 indicate intermediate ratings.

For example, the word "thief" may be rated highly whereas the word "grape" may be rated low.

There are no right or wrong answers - it's up to you to decide. There are quite a few words, so don't spend a lot of time on each one. Just go with your first judgment.

A number line will appear on-screen for you to use in making your rating. Please use the rating options displayed below the word to indicate your choice.

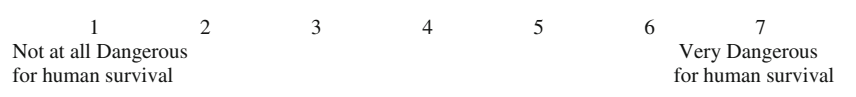

\section{Written instructions for the usefulness rating task}

We would like you to rate how useful for human survival you think each word's referent is.

Your ratings will be made on a 1 to 7 scale, a value of 1 indicating that the referent is not at all useful to human survival and a value of 7 indicating that the referent is extremely useful for human survival. Values of 2 to 6 indicate intermediate ratings.

For example, the word "grape" may be rated highly whereas the word "ash" may be rated low.

There are no right or wrong answers-it's up to you to decide. There are quite a few words, so don't spend a lot of time on each one. Just go with your first judgment.

A number line will appear on-screen for you to use in making your rating. Please use the rating options displayed below the word to indicate your choice.

$\begin{array}{ccccccc}1 & 2 & 3 & 4 & 5 & 6 & \begin{array}{c}7 \\ \text { Not at all Useful } \\ \text { for human survival }\end{array}\end{array}$

\section{References}

Amsel, B. D., Urbach, T. P., \& Kutas, M. (2012). Perceptual and motor attribute ratings for 559 object concepts. Behavior Research Methods, 44, 1028-1041. https://doi.org/10.3758/s13428-012-0215-z

Balota, D. A., Yap, M. J., Cortese, M. J., Hutchison, K. A., Kessler, B., Loftis, B., ... Treiman, R. (2007). The English Lexicon Project. Behavior Research Methods, 39, 445-459. https://doi.org/10.3758/ BF03193014

Barsalou, L. W. (2008). Grounded cognition. Annual Review of Psychology, 59, 617-645. https://doi.org/10.1146/annurev.psych. 59.103006 .093639

Bennett, S. D. R., Burnett, A. N., Siakaluk, P. D., \& Pexman, P. M. (2011). Imageability and body-object interaction ratings for 599 multisyllabic nouns. Behavior Research Methods, 43, 1100-1109. https://doi.org/10.3758/s13428-011-0117-5 
Bonin, P., Gelin, M., \& Bugaiska, A. (2014). Animates are better remembered than inanimates: Further evidence from word and picture stimuli. Memory \& Cognition, 42, 370-382. https://doi.org/10.3758/ s13421-013-0368-8

Borghi, A. M., \& Cimatti, F. (2010). Embodied cognition and beyond: Acting and sensing the body. Neuropsychologia, 48, 763-773.

Boronat, C. B., Buxbaum, L. J., Coslett, H. B., Tang, K., Saffran, E. M., Kimberg, D. Y., \& Detre, J. A. (2005). Distinctions between manipulation and function knowledge of objects: Evidence from functional magnetic resonance imaging. Cognitive Brain Research, 23, 361373. https://doi.org/10.1016/j.cogbrainres.2004.11.001

Brodeur, M. B., Dionne-Dostie, E., Montreuil, T., \& Lepage, M. (2010). The Bank of Standardized Stimuli (BOSS), a new set of 480 normative photos of objects to be used as visual stimuli in cognitive research. PLoS ONE, 5, e10773. https://doi.org/10.1371/journal.pone. 0010773

Brysbaert, M., \& New, B. (2009). Moving beyond Kučera and Francis: A critical evaluation of current word frequency norms and the introduction of a new and improved word frequency measure for American English. Behavior Research Methods, 41, 977-990. https://doi.org/10.3758/BRM.41.4.977

Connell, L., \& Lynott, D. (2016). Embodied semantic effects in visual word recognition. In Y. Coello \& M. Fischer (Eds.), Foundations of embodied cognition: Vol. 2. Conceptual and interactive embodiment (pp. 71-92). Hove, UK: Psychology Press.

Creem, S. H., \& Proffitt, D. R. (2001). Grasping objects by their handles: A necessary interaction between cognition and action. Journal of Experimental Psychology: Human Perception and Performance, 27, 218-228. https://doi.org/10.1037/0096-1523.27.1.218

Díez-Álamo, A. M., Díez, E., Alonso, M. Á., Vargas, C. A., \& Fernandez, A. (2017). Normative ratings for perceptual and motor attributes of 750 object concepts in Spanish. Behavior Research Methods. Advance online publication. https://doi.org/10.3758/s13428-0170970-y

Garcea, F. E., \& Mahon, B. Z. (2012). What is in a tool concept? Dissociating manipulation knowledge from function knowledge. Memory \& Cognition, 40, 1303-1313.

Glenberg, A. M. (2015). Few believe the world is flat: How embodiment is changing the scientific understanding of cognition. Canadian Journal of Experimental Psychology, 69, 165-171.

Grèzes, J., \& Decety, J. (2002). Does visual perception of object afford action? Evidence from a neuroimaging study. Neuropsychologia, $40,212-222$

Guérard, K., Lagacé, S., \& Brodeur, M. B. (2015). Four types of manipulability ratings and naming latencies for a set of 560 photographs of objects. Behavior Research Methods, 47, 443-470. https://doi.org/ 10.3758/s13428-014-0488-5

Hansen, D., Siakaluk, P. D., \& Pexman, P. M. (2012). The influence of print exposure on the body-object interaction effect in visual word recognition. Frontiers in Human Neuroscience, 6, 113. https://doi. org/10.3389/fnhum.2012.00113

Hargreaves, I. S., Leonard, G. A., Pexman, P. M., Pittman, D. J., Siakaluk, P. D., \& Goodyear, B. G. (2012). The neural correlates of the bodyobject interaction effect in semantic processing. Frontiers in Human Neuroscience, 6, 22. https://doi.org/10.3389/fnhum.2012.00022

Hargreaves, I. S., White, M., Pexman, P. M., Pittman, D., \& Goodyear, B. G. (2012). The question shapes the answer: The neural correlates of task differences reveal dynamic semantic processing. Brain and Language, 120, 73-78.

Hauk, O., Johnsrude, I., \& Pulvermüller, F. (2004). Somatotopic representation of action words in human motor and premotor cortex. Neuron, 41, 301-307. https://doi.org/10.1016/S0896-6273(03) 00838-9

Inkster, M., Wellsby, M., Lloyd, E., \& Pexman, P. M. (2016). Development of embodied word meanings: Sensorimotor effects in children's lexical processing. Frontiers in Psychology, 7, 317. https://doi.org/10.3389/fpsyg.2016.00317

Just, M. A., Cherkassky, V. L., Aryal, S., \& Mitchell, T. M. (2010). The neurosemantic theory of concrete noun representations based on the underlying brain codes. PLoS ONE, 5, e8622. https://doi.org/10. 1371/journal.pone.0008622

Kang, S. H. K., Yap, M. J., Tse, C.-S., \& Kurby, C. A. (2011). Semantic size does not matter: "Bigger" words are not recognized faster. Quarterly Journal of Experimental Psychology, 64, 1041-1047.

Kuperman, V., Stadthagen-Gonzalez, H., \& Brysbaert, M. (2012). Age-of acquisition ratings for 30,000 English words. Behavior Research Methods, 44, 978-990. https://doi.org/10.3758/s13428-012-0210-4

Lagacé, S., Downing-Doucet, F., \& Guérard, K. (2013). Norms for grip agreement for 296 photographs of objects. Behavior Research Methods, 45, 772-781.

Madan, C. R. (2014). Manipulability impairs association-memory: Revisiting effects of incidental motor processing on verbal pairedassociates. Acta Psychologica, 149, 45-51. https://doi.org/10.1016/ j.actpsy.2014.03.002

Madan, C. R. (2017). Motivated cognition: Effects of reward, emotion, and other motivational factors across a variety of cognitive domains. Collabra: Psychology, 3, 24.

Madan, C. R., Chen, Y. Y., \& Singhal, A. (2016). ERPs differentially reflect automatic and deliberate processing of the functional manipulability of objects. Frontiers in Human Neuroscience, 10, 360. https://doi.org/10.3389/fnhum.2016.00360

Madan, C. R., Ng, A., \& Singhal, A. (2018). Prototypical actions with objects are more easily imagined than atypical actions. Journal of Cognitive Psychology, 30, 314-210.

Madan, C. R., \& Singhal, A. (2012). Encoding the world around us: Motor-related processing influences verbal memory. Consciousness and Cognition, 21, 1563-1570.

Magnié, M. N., Besson, M., Poncet, M., \& Dolisi, C. (2003). The Snodgrass and Vanderwart set revisited: Norms for object manipulability and for pictorial ambiguity of objects, chimeric objects, and nonobjects. Journal of Clinical and Experimental Neuropsychology, 25, 521-560. https://doi.org/10.1076/jcen.25.4.521.13873

Mahon, B. Z. (2015). The burden of embodied cognition. Canadian Journal of Experimental Psychology, 69, 172-178. https://doi.org/ $10.1037 /$ cep0000060

Montefinese, M., Ambrosini, E., Fairfield, B., \& Mammarella, N. (2013). The "subjective" pupil old/new effect: Is the truth plain to see? International Journal of Psychophysiology, 89, 48-56.

Nairne, J. S. (2010). Adaptive memory: Evolutionary constraints on remembering. In B. H. Ross (Ed.), The psychology of learning and motivation (Vol. 53, pp. 1-32). Burlington, VT: Academic Press.

Pexman, P. M. (2012). Meaning-level influences on visual word recognition. In J. S. Adelman (Ed.), Visual word recognition: Vol. 2. Meaning and context, individuals and development (pp. 24-43). New York, NY: Psychology Press.

Pexman, P. M., Heard, A., Lloyd, E., \& Yap, M. J. (2017). The Calgary Semantic Decision Project: Concrete/abstract decision data for 10, 000 English words. Behavior Research Methods, 49, 407-417. https://doi.org/10.3758/s13428-016-0720-6

Pexman, P. M., \& Yap, M. J. (2018). Individual differences in semantic processing: Insights from the Calgary Semantic Decision Project. Journal of Experimental Psychology: Learning, Memory, and Cognition. https://doi.org/10.1037/xlm0000499

Phillips, C. I., Sears, C. R., \& Pexman, P. M. (2012). An embodied semantic processing effect on eye gaze during sentence reading. Language and Cognition, 4, 99-114.

Proverbio, A. M., Del Zotto, M., \& Zani, A. (2007). The emergence of semantic categorization in early visual processing: ERP indices of animal vs. artifact recognition. BMC Neuroscience, 8,24 . https:// doi.org/10.1186/1471-2202-8-24 
Salmon, J. P., Matheson, H. E., \& McMullen, P. A. (2014). Slow categorization but fast naming for photographs of manipulable objects. Visual Cognition, 22, 141-172.

Salmon, J. P., McMullen, P. A., \& Filliter, J. H. (2010). Norms for two types of manipulability (graspability and functional usage), familiarity, and age of acquisition for 320 photographs of objects. Behavior Research Methods, 42, 82-95. https://doi.org/10.3758/BRM.42.1. 82

Sereno, S. C., O’Donnell, P. J., Sereno, M. E. (2009). Size matters: Bigger is faster. Quarterly Journal of Experimental Psychology, 62, 11151122 .

Shebani, Z., \& Pulvermüller, F. (2013). Moving the hands and feet specifically impairs working memory for arm- and leg-related action words. Cortex, 49, 222-231.

Siakaluk, P. D., Pexman, P. M., Aguilera, L., Owen, W. J., \& Sears, C. R. (2008). Evidence for the activation of sensorimotor information during visual word recognition: The body-object interaction effect. Cognition, 106, 433-443. https://doi.org/10.1016/j.cognition.2006. 12.011

Siakaluk, P. D., Pexman, P. M., Sears, C. R., Wilson, K., Locheed, K., \& Owen, W. J. (2008). The benefits of sensorimotor knowledge: Body-object interaction facilitates semantic processing. Cognitive Science, 32, 591-605. https://doi.org/10.1080/03640210802035399

Taikh, A., Hargreaves, I. S., Yap, M., \& Pexman, P. M. (2014). Semantic classification of pictures and words. Quarterly Journal of Experimental Psychology, 68, 1502-1518.

Thill, S., \& Twomey, K. E. (2016). What's on the inside counts: A grounded account of concept acquisition and development. Frontiers in Psychology, 7, 402. https://doi.org/10.3389/fpsyg. 2016.00402

Tillotson, S. M., Siakaluk, P. D., \& Pexman, P. M. (2008). Body-object interaction ratings for 1,618 monosyllabic nouns. Behavior Research Methods, 40, 1075-1078. https://doi.org/10.3758/BRM. 40.4.1075
Tobia, M. J., \& Madan, C. R. (2017). Tool selection and the ventral-dorsal organization of tool-related knowledge. Physiological Reports, 5, e13078.

Tousignant, C., \& Pexman, P. M. (2012). Flexible recruitment of semantic richness: Context modulates body-object interaction effects in lexical-semantic processing. Frontiers in Human Neuroscience, 6, 53. https://doi.org/10.3389/fnhum.2012.00053

van Elk, M. (2014). The left inferior parietal lobe represents stored handpostures for object use and action prediction. Frontiers in Psychology, 5, 333. https://doi.org/10.3389/fpsyg.2014.00333

Van Havermaet, L. R., \& Wurm, L. H. (2014). Semantic effects in word recognition are moderated by body-object interaction. Mental Lexicon, 9, 1-22.

Van Havermaet, L. R., \& Wurm, L. H. (2017). Effects of danger, usefulness, and body-object interaction in picture naming. Mental Lexicon, 12, 51-70.

Wagenmakers, E.-J. (2007). A practical solution to the pervasive problem of $p$ values. Psychonomic Bulletin \& Review, 14, 779-804. https:// doi.org/10.3758/BF03194105

Wellsby, M., \& Pexman, P. M. (2014). The influence of bodily experience on children's language processing. Topics in Cognitive Science, 6 , 425-441.

Wurm, L. H. (2007). Danger and usefulness: An alternative framework for understanding rapid evaluation effects in perception? Psychonomic Bulletin \& Review, 14, 1218-1225. https://doi.org/ 10.3758/BF03193116

Wurm, L. H., \& Seaman, S. R. (2008). Semantic effects in naming and perceptual identification, but not in delayed naming: Implications for models and tasks. Journal of Experimental Psychology: Learning, Memory, and Cognition, 34, 381-398.

Yap, M. J., Pexman, P. M., Wellsby, M., Hargreaves, I. S., \& Huff, M. J. (2012). An abundance of riches: Cross-task comparisons of semantic richness effects in visual word recognition. Frontiers in Human Neuroscience, 6, 72. https://doi.org/10.3389/fnhum.2012.00072 